\title{
Plasma homocysteine and oxidative stress in cardiovascular disease
}

\author{
S.S. Moselhy ${ }^{\mathrm{a}}$ and S.H. Demerdash ${ }^{\mathrm{b}}$ \\ ${ }^{a}$ Biochemistry Department, Faculty of Science, Ain Shams University, Cairo, Egypt \\ ${ }^{\mathrm{b}}$ Cardiology Department, Faculty of Medicine Ain Shams University, Cairo, Egypt
}

\begin{abstract}
Hyperhomocysteinemia (Hhcy) has been associated with pathological and stressfull conditions and is a risk factor for cardiovascular disease (CVD). The aim of this study was to evaluate the correlation between plasma homocysteine (hcy) and lipid peroxidation in patient with CVD. This study was carried out on 40 patients with CVD as well as 15 healthy volunteers of comparable age and gender as control group. The patients were divided into 2 groups as follows: group I, included 20 patients with acute myocardial infarction and group II, included 20 patients with atherosclerotic coronary artery disease with no evidence of previous myocardial infarction . Plasma hcy, nitric oxide (NO) and malondialdhyde (MDA) [as index of lipid peroxidation] were measured in all groups. In addition serum total-cholesterol, HDL, LDL and triglycerides were evaluated. Results obtained showed that, there was a significant elevation in the levels of plasma hcy, NO and MDA in groups I and II as compared to control group. There was a strong positive correlation between plasma hcy and MDA $(r=0.59, p<0.001)$. Also NO was positively correlated with both hey $(r=0.49, p<0.001)$ and MDA $(r=0.51, p<0.001)$. Serum total cholesterol, LDL, and triglycerids were also significantly elevated while serum HDL was significantly decreased in groups I and II as compared to control group. It can be concluded that, hyperhomocysteinemia is a possible factor in free radical generation and therefore cardiovascular diseases.
\end{abstract}

\section{Introduction}

Oxidative stress occurs when there is an imbalance between free radical production and antioxidant capacity. This may be due to increased free radical formation in the body and/or loss of normal antioxidant defense, this disturbance has been associated with the development of cardiovascular disease (CVD) [29]. CVD and cerbrovascular disease is the leading cause of death [18]. Atherosclerosis of coronary arteries is responsible for almost all cases of CVD [12]. The identification of risk factors of atherosclerotic lesions leads to development of strategies for prevention of CVD and its complications. Conventional risk factors for coronary artery disease including hyperlipidemia, smoking, hypertension and diabetes are all associated with increased oxidative stress due to excess free radical activity in the vascular wall. This may facilitate the development of vascular disease [8].

Homocysteine (hcy) is a thiol containing amino acid produced by demethylation of methionine. Half of the hcy formed goes through the trans-sulphuration path- way and the other half takes a methyl group from betaine or 5-methyltetrahydrofolic acid. Methionine synthesis is a cobalamin dependent enzyme which is functionally impaired when vitamin B12 metabolism is abnormal [32].

The nitric oxide (NO) is increasingly recognized as an important intra-and intercellular messenger [4]. The $\mathrm{NO}$ is identical to endothelium-derived relaxing factor and is the principle signal for relaxation of vascular smooth muscle cells [27].The enzyme nitric oxide synthase converts L-argnine into citrulline and NO [22]. The role of NO has been implicated in a variety of biological processes such as neurotransmission, tumor cell killing, immunity, and inflammatory processes [15].

Experimental studies revealed that, infusion of hcy into animals results in damage of vascular endothelium and reduced platelet survival [16]. Hyperhomocysteinemia (Hhcy) has been associated with pathological and stressful conditions and is a risk factor for CVD [10]. This study was designed to evaluate the correlation among plasma hcy, lipid peroxidation indicator (MDA) and NO in patients with cardiovascular disease. 


\section{Subjects and methods}

\section{1. a) subjects}

This study was carried out on 40 patients with CVD as well as 15 healthy volunteers of comparable age and gender as control group. The patients were divided into two groups as follows: Group I; included 20 patients (14 males and 6 females aged 42-60 years) with acute myocardial infarction and; group II; included 20 patients (12 males and 8 females aged 40-58 years) with coronary atherosclerosis with no evidence of previous myocardial infarction. The patients were diagnosed at the cardiology department. Ain Shams University Hospital. Patients with diabetes mellitus, renal impaired function were excluded from this study.

All subjects (patients and control) were subjected to clinical examination, echocardiographic imaging, electrocardiography, angiographically and treadmill exercise stress test. Diagnosis of myocardial infarction was based on the presence of two or more of the following criteria (i) typical anginal chest pain lasting for 30 minutes or more. (ii) presence of ST segment elevation in resting electrocardiography. (iii) elevation of the cardiac enzymes (creatine kinase [CK] or CK,MB) twice or more the upper normal level. Meanwhile patients with coronary atherosclerosis were chosen from patients attending the cardiac catheterization laboratory for coronary angiography. The diagnosis was made when there was $50 \%$ or more luminal narrowing of one or more of the measure coronary areteries. Fasting venous blood samples were taken on the second day of attack, plasma and serum were separated and kept at $-70^{\circ} \mathrm{C}$ till analysis.

\section{2. b) Methods}

1. Plasma homocysteine was assayed by ELISA, as described in Frantzen et al. [13].

2. Plasma lipid peroxidation indicator, malondialdhyde (MDA) was evaluated by thiobarbituric acid according to Zima et al. [41].

3. Plasma nitrite and nitrate were assayed by the modified microassay as described by Vodovotz [36]. Briefly, nitrate in plasma was reduced to nitrite by Cadmium and assayed by Griess reagents.

4. Lipids profile was evaluated including, total cholesterol [17], HDL-cholesterol [24], LDLcholesterol [14] and triglycerides [37].
Results are expressed as Mean \pm SD. The mean, standard deviation and $\mathrm{p}$ values were performed using the statistical package for the social science (Spss) whereas the correlation coefficient was performed using excell 5 on IBM, PC computer.

\section{Results}

Table 1, showed that, plasma levels of hcy, MDA and NO were significantly elevated in patients with acute myocardial infarction and coronary artery atherosclerosis $(p<0.001,0.001$ and 0.001$)$ as compared to control subjects. A statistically positive correlation was obtained between hcy and MDA $(r=0.59, p<0.001)$. Also, plasma NO level revealed statistically significant positive correlation with plasma MDA $(r=0.59$, $P<0.001)$ and plasma hcy $(r=0.5, p<0.001)$.Lipid profile results revealed that, serum total cholesterol, LDL and triglycerides were significantly elevated while HDL was significantly decreased in groups I and II as compared to healthy subjects(the $\%$ changes were $44 \%$, $54 \%$ for total cholesterol, $57.2 \%, 69 \%$ for LDL , 63\%, $82.5 \%$ for triglycerides and $-26.4 \%,-31 \%$ for HDL respectively as compared to control group (Table 2 ).

\section{Discussion}

Traditional risk factors for coronary artery disease (CAD) such as hypertension, smoking and diabetes mellitus are all associated with increased oxidative stress due to excess free radical activity in the vascular wall. This may facilitate the development of vascular disease because of increased oxidation of LDLparticles which increases propensity to deposition in the vascular wall [25].

Epidemiological studies have consistently demonstrated that, high plasma hcy is an independent risk factor for atherosclerosis, as important as serum cholesterol level [9]. However, unlike hypercholesterolemia, hyperhomocysteinemia (Hhcy) is not a sufficient stimulus for the development of atherosclerosis but predisposes to complications and progression of the arterial lesion [3]. In addition, Hhcy is common in patients with peripheral arterial occlusive disease, coronary heart disease, cerebrovascular disease, carotid artery stenosis and venous thromboembolism [33]. This study aimed to evaluate the correlation between plasma hcy and oxidative stress in patients with cardiovascular diseases (CVD). The results obtained showed that there was a 
Table 1

Plasma levels of homocystiene (hcy), lipid peroxidation indicator (MDA)and nitric oxide (N0)in all studied groups (Mean \pm . SD)

\begin{tabular}{lccc}
\hline Groups & $\begin{array}{c}\text { Plasma hcy } \\
\text { umol/L }\end{array}$ & $\begin{array}{c}\text { Plasma MDA MDA } \\
\text { umol/L }\end{array}$ & $\begin{array}{c}\text { Plasma NO } \\
\text { umol/L }\end{array}$ \\
\hline $\begin{array}{l}\text { Control group } \\
(n=15)\end{array}$ & - & \\
Range - & $(4.5-14.2)$ & $(0.5-2.9)$ & $(8.1-16.1)$ \\
Mean \pm SD & $8.69 \pm 2.7$ & $1.32 \pm 0.7$ & $11.46 \pm 2.6$ \\
Group I & & & \\
Acute myocardial infarction & & & \\
$(n=20)$ & $(10.1-27)$ & $(1.2-5.9)$ & $(13-22.1)$ \\
Range & $21.6 \pm 4.3$ & $2.42 \pm 1.08$ & $17.69 \pm 2.57$ \\
Mean \pm SD & $<0.001$ & $<0.001$ & $<0.001$ \\
P value & $148.5 \%$ & $83.3 \%$ & $54.3 \%$ \\
$\%$ change & & & \\
Group II & & & \\
Atherosclerosis & & & \\
$(n=20)$ & $(9.3-22)$ & $(1-4.1)$ & $(10.5-19.1)$ \\
Range & $16.91 \pm 3.3$ & $1.98 \pm 1.0$ & $15.1 \pm 2.46$ \\
Mean \pm SD & $<0.001$ & $<0.05$ & $<0.001$ \\
P value & $94.4 \%$ & $50 \%$ & $32.45 \%$ \\
$\%$ change & $\%$ & & \\
\hline
\end{tabular}

$\mathrm{P}$ value compared to control. \% change between groups I\& II and control.

Table 2

Serum total cholesterol HDL-Cholesterol, LDL-Cholesterol and triglycerides in all studied groups (Mean $\pm \mathrm{SD})$

\begin{tabular}{|c|c|c|c|c|}
\hline Groups & $\begin{array}{c}\text { Total cholesterol } \\
\mathrm{mg} / \mathrm{dl}\end{array}$ & $\begin{array}{c}\text { HDL-Cholesterol } \\
\mathrm{mg} / \mathrm{dl}\end{array}$ & $\begin{array}{l}\text { LDL-Cholesterol } \\
\mathrm{mg} / \mathrm{dl}\end{array}$ & $\begin{array}{c}\text { Triglycerides } \\
\text { mg/dl }\end{array}$ \\
\hline \multicolumn{5}{|c|}{$\begin{array}{l}\text { Control group } \\
(n=15)\end{array}$} \\
\hline Range & $(166-216)$ & $(40.1-52.3)$ & $(90-135)$ & $(95.6-148.3)$ \\
\hline Mean \pm SD & $191.5 \pm 18.6$ & $42.8 \pm 3.2$ & $106.6 \pm 18.9$ & $130.9 \pm 17$ \\
\hline \multicolumn{5}{|c|}{ Group I } \\
\hline \multicolumn{5}{|c|}{$\begin{array}{l}\text { Acute myocardial infarction } \\
(n=20)\end{array}$} \\
\hline Range & $(190-350)$ & $(26-37)$ & $(110-240)$ & $(120-244)$ \\
\hline Mean $\pm \mathrm{SD}$ & $277.2 \pm 38$ & $31.5 \pm 3.3$ & $167.6 \pm 37.5$ & $212 \pm 65$ \\
\hline$P$ value & $<0.001$ & $<0.001$ & $<0.001$ & $<0.001$ \\
\hline$\%$ change & $44 \%$ & $-26.4 \%$ & $57.2 \%$ & $63 \%$ \\
\hline \multicolumn{5}{|c|}{ Group II } \\
\hline \multicolumn{5}{|c|}{$\begin{array}{l}\text { Atherosclerosis } \\
(n=20)\end{array}$} \\
\hline Range & $(199-353)$ & $(25.2-34.3)$ & $(115-260)$ & $(160-268)$. \\
\hline Mean \pm SD & $295.21 \pm 38$ & $29.4 \pm 1.9$ & $180.9 \pm 41$ & $238 \pm 91$ \\
\hline$P$ value & $<0.001$ & 0.001 & $<0.001$ & 0.001 \\
\hline$\%$ change & $54.1 \%$ & $-31 \%$ & $69.6 \%$ & $82.5 \%$ \\
\hline
\end{tabular}

P value compared to control. \% change between groups I \& II and control.

highly significant elevation in the levels of plasma hcy, MDA and NO in patients with either acute myocardial infarction or coronary artery atherosclerosis as compared to healthy ones (The \% changes were $148.5 \%$ and $94.4 \%$ for hcy, $83.3 \%$ and $50 \%$, for MDA, $54.3 \%$ and $32.45 \%$ for NO respectively). The positive correlation between plasma hcy and lipid peroxidation in this study may suggest the possible role of hcy in the release of reactive oxygen species. Wei and Quast [38] found that, there was an excessive glutamate excitotoxicity, leading to enhanced generation of hydroxylradicals via a NO-mediated mechanism and resulting in severe ischemic injury. Mc Murry et al. [26] reported that, thiobarbituric acid reactive substances are longer lived products of lipid peroxidation that are increased in oxidative stress in heart failure and CAD but are known to be non specific. Protective effects of NO have been reported during cerebal and myocardial is- 
chemia. These effects of $\mathrm{NO}$ are most probably indirect effects of $\mathrm{NO}$ as a consequence of its vasodilatory activity to increase the blood flow, its capability to inhibit adhesion of lymphocytes, monocytes, and neutrophils to the endothelium (thereby decreasing local $\mathrm{O}_{2}^{--}$generation), and its ability to inhibit platelets aggregation, thus decreasing capillary occlusions [23].

In vitro studies, hcy may acutely induce oxidative stress by autoxidation of sulfhydryl group of homocysteine to generate hydrogen peroxide [31], by decreasing intracellular antioxidant defense (glutathione and glutathione peroxidase) [34] and by direct cytotoxic effects (in cultured endothelial cells) [2]. Cavalca et al. [7] evaluated the possible role of hcy in inducing oxidative stress in CAD, they reported that plasma hcy and MDA concentrations were significantly higher in CVD patients than in control.

Zhang et al. [40] reported that the vasodilator effect of NO is attenuated in the presence of hcy, also the infusion of hcy with copper inhibits NO related vasodilatory responses by scavenging of NO. This may be one of the mechanisms by which Hhcy predisposes to CVD. The significant elevation of NO level in this study could be a protective mechanism against Hhcy to produce S-nitrosohomocysteine, which is less injurious than hcy [20]. In vitro study, NO decreases hcy by its conversion to the vasodialative and antioxidant compound S-nitrosohomocysteine [6].

Khan et al. [19] reported that, the significant elevation of NO might reflect the elaboration of the angiopathic free radical peroxynitril through the generation of superoxide anions and hydrogen peroxide. Hhcy has been associated with premature atherothrombotic vascular disease. The pathophysiological mechanisms linking Hhcy to vascular disease have been extensively studied by Van Guldener and Stehouwer [35], they suggested that, hcy limits the bioavailability of NO, increases oxidative stress, stimulates smooth cell proliferation and alters elastic wall properties.

Experimental studies by Kitiyakara et al. [21] revealed that, oxidative stress and Hhcy culminate in abnormal vascular and endothelial regulation, functional NO deficiency, vascular hypertrophy and atherosclerosis. Welch et al. [39] hypothesized that, hcy contributes to atherosclerosis by affecting cytokine induced production of NO by vascular smooth muscle cells. Also the authors found that, inducible nitric oxide synthase (iNOS) activity and iNOS protein level were increased significantly in the hcy treated cells as compared to control.

Hyperhomocystenima-induced atherosclerosis is probably due to various factors, including endothelial cell injury, inability to sustain S-nitrosohomocysteine formation because of imbalance between production of NO by endothelial cells and hcy, smooth muscle proliferation and thromboembolism [28].

Lipoproteins abnormalities play a critical role in atherogensis and CAD, such as elevated LDL, reduced HDL, increased triglycerides rich lipoproteins (VLDL and intermediate density lipoprotein iDL) and increased lipoprotein (a) [1].

Table 2 showed that, there were a highly significant elevation in the levels of serum total-cholesterol, LDL and triglycerides, while serum HDL was significantly decreased in groups I and II as compared to control group (The $\%$ changes were $44 \%, 54.5 \%$ for total cholesterol, $37.2 \%, 69 \%$ for LDL, $63 \%, 82.5 \%$ for triglyecrides and $-26.4 \%,-31 \%$ for HDL respectively)

Study carried out by Dornner et al. [11] showed that, male patients with coronary heart disease had higher VLDL, triglycerides and low HDL than control while female patients did not have any significant difference in lipoproteins versus the controls.

Qujeg et al. [30] stated that, serum total hcy levels were significantly correlated with LDL-cholesterol $(P<0.05, r=0.98)$ and HDL- cholesterol $(P<$ 0.05, $r=0.98)$. Brouwer et al. [5] found that, male CAD patients had higher cholesterol, triglycerides, LDL-cholesterol, apo-B and decreased HDLcholesterol and HDL/cholesterol concentration. Other risk factors were increased lipoprotein(a), Hhcy, renal disease and diabetes mellitus. We can concluded that plasma MDA, NO and hcy levels can be used as prognostic markers in patients with CVD.

\section{References}

[1] C.M. Ballantyne, Current thinking in lipid lowering, Am J Med 104(6A) (1998), 33S-41S.

[2] G. Blundell, B.G. Jones, F.A. Rose and N. Tudball, Homocysteine mediated endothelial cell toxicity and its amelioration, Atherosclerosis 122 (1996), 163-172.

[3] C.J. Boushy, S.A. Beresford, G.S. Omenn and A.G. Motulsky, A quantitative assessment of plasma homocysteine as a risk factor for vascular disease probable benefits of increasing folic acid intake, J Am Med Assoc 274 (1995), 1049-1057.

[4] D.S. Bredt and S.H. Snyder, Nitric oxide: A physiologic messenger molecule, Ann Rev Biochem 63 (1994), 175-195.

[5] D.A. Brouwer, C.B. Leerink, H.N. Steward, T.A. Kroon, G.B. Suverkropp, J.W. Romer, M. Volmer and F.A. Muskiet, Lipids, lipoprotein E genotypes and other risk factors of patients with coronary artery disease in Curacao, West indian Med J 46(2) (1997), 47-52. 
[6] F.M. Cassone, O. Laurenti, G. Desideri, M.C. Bravi, O. De luca, M.C. Marinucci, G. De Mattia and C. Ferri, L-argnine infusion decreases plasma total homocysteine concentrations through increased nitric oxide production and decreased oxidative status in type II diabetic patients, Diabetologia $\mathbf{4 5}(8)$ (2002), 1120-1127.

[7] V. Cavalca, G. Cighetti, F. Bamonti, A. loaldi, L. Bortone, C. Novenbrine, M. De franceschi, R. Bclardinelli and M.D. Guazzi, Oxidative stress and homcysteine in coronary artery disease, Clin Chem 47(5) (2001), 887-892.

[8] M.T. Christophe, P. Marcel, C.I. Michele, T. Fawzi, A. MicheL and M.L. Jean-Philippe, Plasma homocysteine and severity of thoracic aortic atherosclerosis, Chest 118 (2000), 1685-1689.

[9] R. Clarke, L. Daly and K. Robison, Hyperhomocysteinemia: An independent risk factor for vascular disease, NEJM 324 (2001), 1149-1155.

[10] A.C. De Olivera, D.C. De Almeida, J.N. Nobrega and S. Tufik, Sleep deprivation reduces total plasma homocysteine levels in rats, Can J Physiol Pharmacol 80(3) (2002), 193-197.

[11] M.G. Donner, G.K. Klein, P.B. Mathes, P. Schwandt and W.O. Richter, Plasma total homocysteine levels in patients with early onset coronary heart disease and a low cardiovacular risk profile, Metabolism 47(3) (1998), 273-279.

[12] M. Elisaf, The treatment of coronary heart disease: An update Part I; An overview of the risk factors for cardiovascular disease, Curr Med Res Opin 17(1) (2001), 18-26.

[13] F. Frantzen, A.L. Faaren, I. Almeim and A.K. Nordhei, An enzyme conversion immunoassay for determining total homocysteine in plasma or serum, Clin. Chem. 44 (1998), 311-316.

[14] W.T. Friedwald, R.L. Levy and D.S. Fredickson, Estimation of the concentration of low denisty lipoprotein cholesterol in plasma,without use of the preparative ultracentrifuge, Clin Chem 18 (1972), 499-502.

[15] N. Gorbunov and E. Esposito, Nitric oxide as a mediator of inflammation [Review], Int J Immunopathol Pharmacol 6 (1993), 67-75.

[16] L.A. Harker, S.L. Slichter, C.R. Scott and R. Ross, Homocysteinemia: Vascular injury and arterial thrombosis, $N$ Eng $J$ Med 291 (1974), 537-543.

[17] R.I. Henary, D.C. Cannon and J.W. Winkelman, Clinical chemistry. principle and techniques, 2nd ed., pp. 1440.

[18] W.F. Ignatius, Emerging relations between infectious diseases and coronary artery disease and atherosclerosis, Canadian Medical Association Journal 163(1) (2000), 49-56.

[19] M.A. Khan, C.S. Thompson, A.M. Emsley, F.H. Mumtaz, D.P. Mikhailidis, G.D. Angelini, R.J. Morgan and J.Y. Jeremy, The interaction of homocysteine and copper markedly inhibits the relaxation of rabbit corpus cavernosum: new risk factor for angiopathic erectile dysfunction? BJU Int 84 (1999), 720724.

[20] W.K. Kim, S-nitrosoation ameliorates homcysteine induced neurotoxicity and calcium responses in primary culture of rat cortical neurons, Neurosci lett 265 (1999), 99-102.

[21] C. Kitiyakara, J. Gonin, Z. Mossy and C.S. Wilcox, Non traditional cardiovascular disease risk factors in end stage renal disease. Oxidative stress and hyperhomocysteinemia, Curr Opin Nephrol Hypertens 5 (2000), 477-487.

[22] R.G. Knowles and S. Moncada, Nitric-oxide synthases in mammals [Review], Biochem J 298 (1994), 249-258.

[23] J. Loscalzo and G. Welsh, Prog. Cardiovasc. Dis. 38 (1995), 87-104.
[24] M.F. Lopes-virella, P. Stone, S. Ellis and J.A. Colwell, Cholesterol determination in high density lipoproteins separated by three different methods, Clin Chem 8 (1977), 882.

[25] S.R. Maxwell, Coronary artery disease-free radical damage, antioxidant protection and the role of homocysteine, Basic Res Cardio 195(1) (2000), 165-171.

[26] J. McMurray, M. Chopra, I. Abdullah, W.E. Smith and H.J. Dargie Evidence of oxidative stress in chronic heart failure in humans, Eur Heart J 14 (1993), 1493-1498.

[27] L.S. Moncada and A. Higgs, The L-argnine-nitric oxide pathway [Review], New Engi J Med 329 (1993), 2002-2012.

[28] K. Prasad, Homocysteine, a risk factor for cardiovascular disease, International Journal of angiology 1 (1999), 76-86.

[29] S. Penckofer, D. Schwertz and K. Florczak, Oxidative stress and cardiovascular diseases in type 2 diabetes: The role of antioxidant and prooxidants, J cardiovasc. Nurs. 16(2) (2002), 68-85.

[30] D. Qujeq, T.S. Omran and L. Hosini, Correlation between total homocysteine, LDL-cholesterol and HDL-cholesterol in the serum of patients with myocardial infarction, Clin Biochem 2 (2001), 97-101.

[31] G. Starkebaum and J.M. Harlan, Endothelial cell injury due to copper -catalyzed hydrogen peroxide generation from homocystiene, J Clin Invest 77 (1986), 1370-1376.

[32] J.J. Sung and J.E. Sanderson, Hyperhomocysteinemia,Helicobacter Pylori. and coronary heart disease, Heart 76 (1996), 305-307.

[33] K. Sydom and R.H. Boger, Homocysteine, endothelial dysfunction and cardiovascular risk: Pathomechanisms and therapeutic options, Z KardioL 90(1) (2001), 1-11.

[34] G.R. Upchurch, G.N. Welch, A.J. Fabian, J.E. Freedman, J.L. Johnson, J.F. Keaney and J. Loscaizo, Homocysteine decreases bioavailable nitric oxide by a mechanism involving glutathione peroxidase, J Biol Chem 272 (1997), 17012-17017.

[35] C. Van Guldener and C.D. Stehouwer, Hyperhomocysteinemia, vascular pathology and endothelial dysfunction, SeminThromb Hemost 26(3) (2000), 81-289.

[36] Y. Vodovotz, Modified microassay for serum nitrite and nitrate, Biotechniques 20(3) (1996), 390-394.

[37] A.W. Wahlefeld, Triglycerides determination after enzymatic hydrolysis, in: Methods of enzymatic analysis, Bergmeyer, ed., 2nd ed, pp. 1440.

[38] J. Wei and M.J. Quast, Effect of nitric oxide synthase inhibitor on a hyperglycemic rat model of reversible focal ischemia; detection of excitatory amino acids release and hydroxyl radical formation, Brain Res 791 (1998), 146-156.

[39] G.N. Welch, G.R. Upchurch, R.S. Farivar, A. Pigazzi, K. Vu, P. Brecher, J.F. Keaney and J. Loscaizo, Homocysteine-induced nitric oxide production in vascular smooth muscle cells by NF-Kappa-B-dependent transcriptional activation of NOS 2, Proc Assoc Am Physicions 110(1) (1998), 22-31.

[40] F. Zhang, A. Slungaard, G.M. Vercellotti and C. Ladecola, Superoxide dependent cerebrovascular effects of homocysteine, Am J physiol 274 (1998), 1704-1711.

[41] T. Zima, S. Stipek, J. Crkovska and J. Platenik, Measurment of lipid peroxidation product-malondialdhyde in biological samples by spectrophotometric and HPLC assay, Klin Bioch Metab 3(24) (1995), 98-102. 


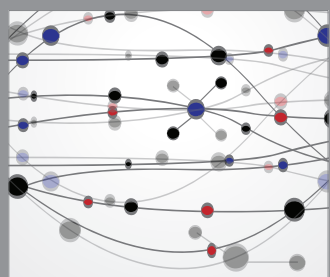

The Scientific World Journal
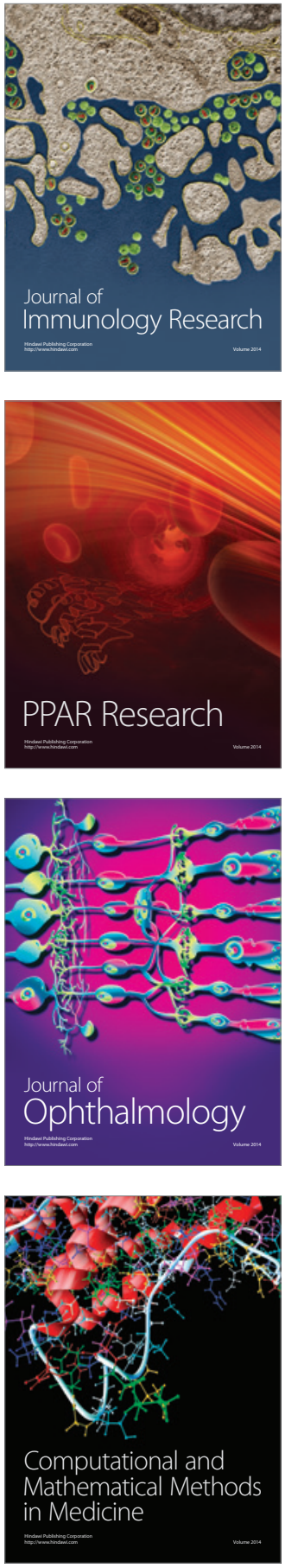

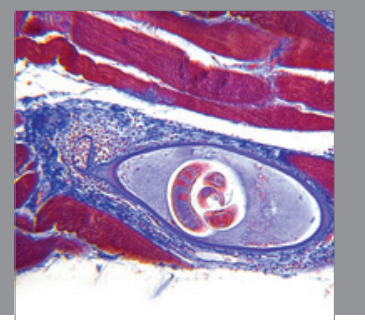

Gastroenterology

Research and Practice
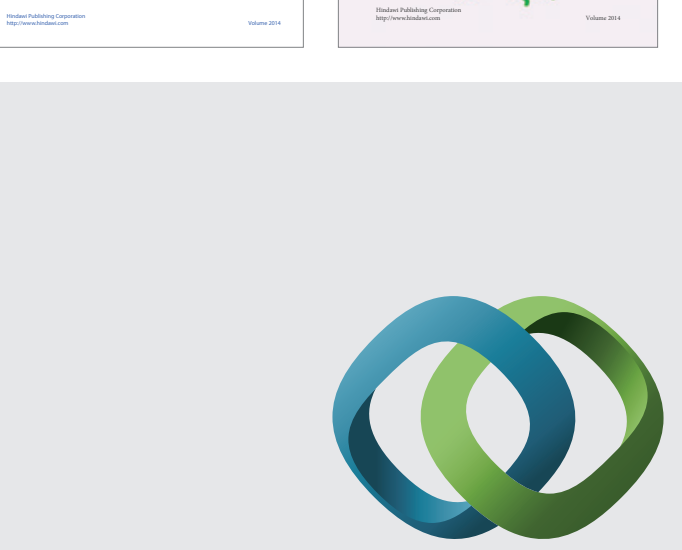

\section{Hindawi}

Submit your manuscripts at

http://www.hindawi.com
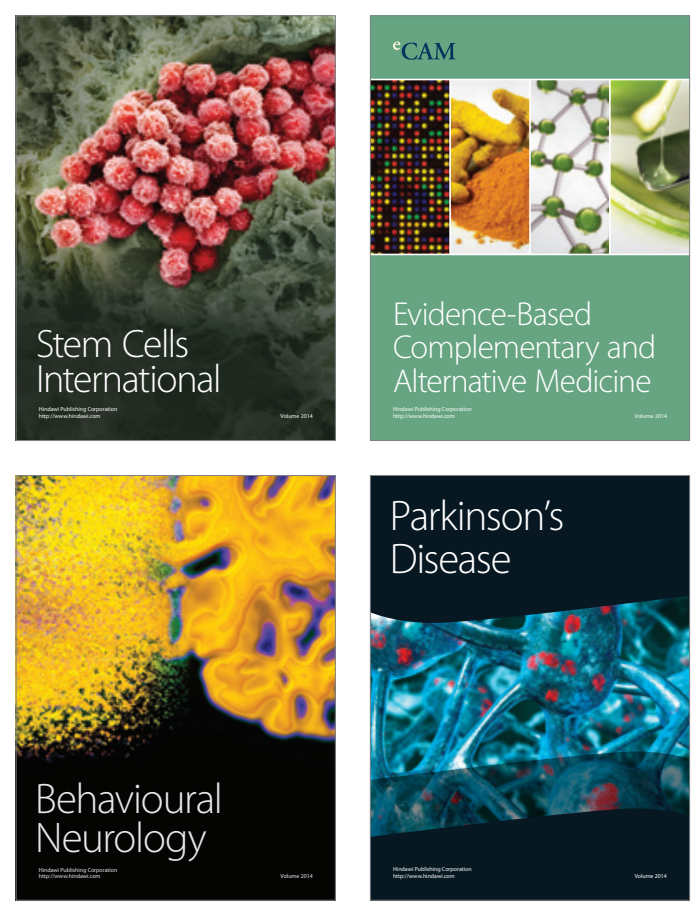

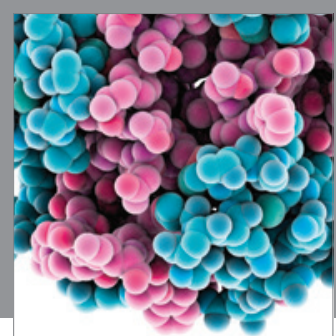

Journal of
Diabetes Research

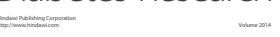

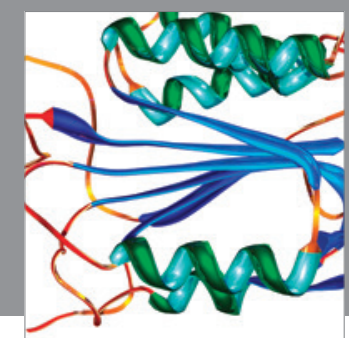

Disease Markers
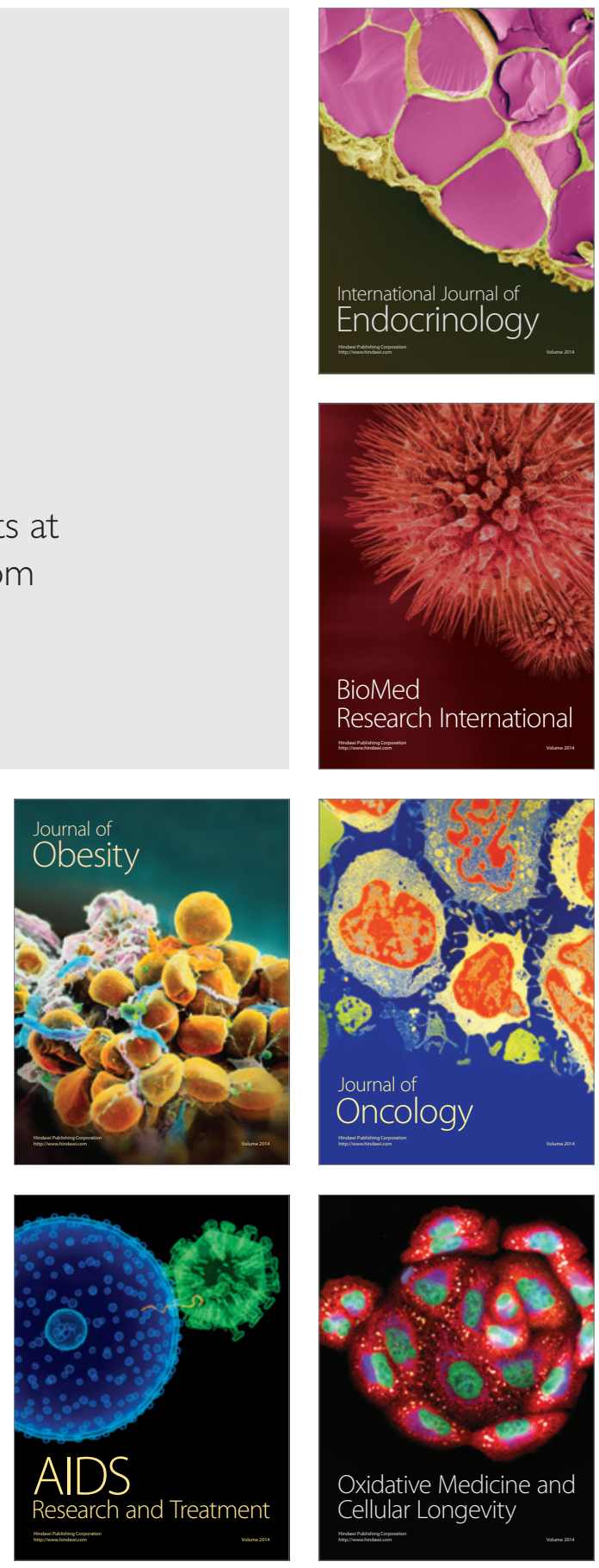